\title{
UTILIZAÇÃO DE UM SOFTWARE PARA A VERIFICAÇÃO DA DERIVADA DE ALGUMAS FUNÇÕES DE RELATIVA COMPLEXIDADE DE DEMONSTRAÇÃO
}

\author{
USE OF SOFTWARE TO CHECK THE DERIVATIVE OF SOME FUNCTIONS \\ WITH DEMONSTRATION COMPLEXITY
}

\author{
Rogeria Teixeira Urzêdo Queiroz ${ }^{1}$ \\ Rhelman Rossano Urzêdo Queiroz²
}

\begin{abstract}
Resumo
O Cálculo Diferencial e Integral é uma disciplina que consta da grade curricular dos períodos iniciais de diversos cursos de graduação. É inegável a importância do conhecimento dos principais conceitos dessa disciplina, bem como ter clareza do seu amplo espectro de aplicações. Entretanto, na opinião de vários professores de Cálculo Diferencial que existe uma grande dificuldade dos alunos ingressantes nos cursos superiores de Ciências Exatas em assimilar os conteúdos propostos nos planos de curso da referida disciplina. Há várias discussões no meio acadêmico relativas a esse tema "dificuldade em Cálculo" e, entre elas, destaca-se a afirmativa de que as deduções de equações e demonstrações de teoremas, com recursos algébricos apenas, aumentam o grau de dificuldade da disciplina. Neste artigo, propõe-se uma forma alternativa de se verificar a derivada de algumas funções, sem os recursos algébricos apresentados pelos livros-texto de Cálculo, com a qual os alunos poderão utilizar a definição de derivada empregando o software Graphmatica.
\end{abstract}

Palavras-chave: Cálculo. Educação Matemática. Softwares educativos.

\footnotetext{
Abstract

Differential and Integral Calculus is a discipline that appears in the initial periods of several undergraduate courses. Therefore, it is undeniable, the importance of knowledge of the main concepts of this discipline, as well as being clear about its wide spectrum of applications. However, it

${ }^{1}$ Mestre em Ensino de Ciências e Matemática, pela PUC Minas. Docente da Fundação Educacional de Mariana E-mail: rogeriatuq@gmail.com

${ }^{2}$ Doutor em Engenharia Metalúrgica e de Minas pela Universidade Federal de Minas Gerais. Docente do Instituto Federal de Minas Gerais - Campus Ouro Preto. E-mail: rhelman.queiroz@ifmg.edu.br
} 
Utilização de um software para a verificação da derivada de algumas funções de relativa complexidade de demonstração

is the opinion of several calculus teachers, that there is a great difficulty for students entering the Universities and Faculties to assimilate the proposed contents of that discipline. There are several discussions in the academic environment related to this theme "difficulty in Calculus" and, among them, the statement that the deductions of equations and theorem demonstrations, with algebraic resources only, increases the degree of difficulty of the discipline. In this work, an alternative way of verifying the derivative of some functions is proposed, without the algebraic resources presented by the compendiums of Calculus, where students can use the definition of derivative, using the software Graphmatica.

Keywords: Differential and Integral Calculus. Mathematical Education. Educational software

\section{Introdução}

O Cálculo Diferencial e Integral foi idealizado de maneira independente pelo inglês Isaac Newton (1643-1727) e pelo alemão Gottfried Wilhelm Leibniz (1646-1716), embora existam discussões polêmicas sobre qual dos dois foi realmente o criador do Cálculo (EVES, 2007, p. 444). Eves (2007) afirma que, por algum tempo depois de Newton e Leibniz, os fundamentos de Cálculo permaneceram obscuros e despercebidos, mas por volta de 1700, a maior parte do Cálculo que hoje se vê nos cursos de graduação já fora estabelecida, juntamente com tópicos mais avançados.

Atualmente, há uma grande aplicabilidade do Cálculo em diversos ramos do conhecimento humano. Dessa forma, o Cálculo Diferencial e Integral figura entre as disciplinas básicas de diversos cursos de graduação (BARROS e MELONI, 2006), o que é compreensivel, pois. Realmente. os conceitos de Cálculo encontram aplicações na Física, como por exemplo, a queda de corpos em um meio material, na transmissão de calor e massa, circuitos elétricos, movimentos de corpos em trajetórias de diversas formas; na Biologia, como no crescimento de uma colônia de 
Utilização de um software para a verificação da derivada de algumas funções de relativa complexidade de demonstração

bactérias; na Química, como na determinação de concentrações de solutos (BRONSON e COSTA, 2006).

Apesar de toda essa importância do Cálculo Diferencial, é de conhecimento de muitos que o ensino e aprendizagem de Cálculo passam por momentos de grande fragilidade e, conforme anunciado por Rezende (2003), muito se tem dito a respeito do "fracasso no ensino de cálculo". Realmente, o Ministério da Educação e Cultura ${ }^{3}$ informa que, no ano de 2000, o índice de reprovação e abandono nos cursos iniciais de Cálculo, nas universidades brasileiras, foi aproximadamente de $80 \%$. São vários os pesquisadores que procuram, em seus estudos, propor soluções que amenizem tal situação (MIRANDA, 2004; CONCEIÇÃO e GONÇALVES, 2003). Para Miranda (2004), deve-se dar uma atenção maior à reestruturação das ementas dos cursos de Cálculo e, também, se pensar em introduzir o Cálculo no Ensino Médio. Conceição e Gonçalves (2003) afirmam que os alunos devem realizar muitos exercícios, principalmente nos conteúdos de derivadas e integrais, para que haja fixação dos conceitos. Porém, há o risco de que o aluno aprenda apenas a repetir regras matemáticas e não consiga entender realmente o conceito ali inserido, que é o mais importante. Para Barros e Meloni (2006), o aluno considera enfadonho, cansativo e sem propósito a repetição continuada de certa prática e complementam afirmando que:

Os exercícios servem para consolidar e automatizar certas técnicas, habilidades e procedimentos necessários para a resolução de problemas, mas, dificilmente podem servir para a aprendizagem e compreensão de conceitos (BARROS e MELONI, 2006, p.1734).

\footnotetext{
$\overline{{ }^{3} \text { http://www.inep.gov }} \cdot$ br/download/censo/2000/Superior/Sinopse_Superior-2000.pdf
} 
Utilização de um software para a verificação da derivada de algumas funções de relativa complexidade de demonstração

Para Gonçalves e Zunchi (2003), as dificuldades relativas ao ensino e à aprendizagem de Cálculo são antigas, pois são encontradas ao longo da história da Matemática. Para esses autores, as dificuldades começam a aparecer desde o conceito intuitivo de limite, ao se trabalhar com números infinitesimais, sendo que, para a maioria dos alunos, é o primeiro contato com esse tópico. Também, dependendo da situação utilizada, com a noção do infinito, por exemplo, aproximar a área de uma figura por $n$ retângulos, principalmente quando $n$ tende a um número muito grande. As primeiras barreiras já começam a surgir neste contexto e se seguirão nos conteúdos seguintes.

Essa situação relacionada ao ensino de Cálculo é grave e, evidentemente, tem levado pesquisadores e professores a desenvolverem métodos de ensino que procurem tornar mais fácil o processo de ensino e aprendizagem da disciplina. Muitas dessas pesquisas utilizam o computador e é justificável, pois nas últimas décadas foi enorme o desenvolvimento de diversos softwares que podem ser aplicados de forma simples e eficiente dentro da sala de aula. Um desses softwares é o Graphmatica (1999) que se constitui em uma ferramenta de plotagem de gráficos, utilizando equações na forma cartesiana ou polar. Também é possivel, com essa ferramenta, o trabalho com conceitos inerentes ao Cálculo, tais como derivadas, integrais, áreas compreendidas entre duas curvas, traçado de uma reta tangente a um ponto de uma curva, bem como a determinação imediata do seu coeficiente angular. Este artigo tem como objetivo geral apresentar um método prático para se verificar, de maneira simples, as derivadas de algumas funções e, como objetivo específico, aplicar a definição de derivada de uma função em um ponto, utilizando-se um método gráfico com o auxílio do software Graphmatica. 
Utilização de um software para a verificação da derivada de algumas funções de relativa complexidade de demonstração

\section{Justificativa}

Trazendo a questão relacionada às dificuldades de ensino e aprendizagem do Cálculo para a região de Mariana e Ouro Preto, elaborou-se um questionário aplicado a um total de 26 alunos do primeiro período de Engenharia de Produção de uma Faculdade de ensino privado da cidade de Mariana. Desse total, $73 \%$ dos alunos são da região de Mariana e Ouro Preto, sendo que $88 \%$ estudaram em escolas públicas estaduais ou federais. Com relação às dificuldades em Matemática, nos cursos fundamental e médio, 54\% apresentaram dificuldades na disciplina. No curso de Cálculo I, verificou-se que $42 \%$ apresentam dificuldades em entender as teorias apresentadas antes de realizar exercícios em sala; $27 \%$ apresentam dificuldades em entender as demonstrações de teoremas e fórmulas e $31 \%$ não entendem a resolução de exercícios. Caso as aulas de Cálculo sejam dadas em laboratórios de Matemática ou Informática, 80\% afirmam que seria possivel amenizar as dificuldades apresentadas e, finalmente, $85 \%$ alegam que a utilização de softwares educativos poderia ajudá-los a entender melhor o conteúdo e sanar as dificuldades apresentadas. Dessa forma, justifica-se a necessidade de se propor novas metodologias de ensino que permitam ao aluno compreender melhor os conceitos apresentados na disciplina de Cálculo.

\section{Metodologia}

Para o desenvolvimento deste trabalho, foram escolhidas, para a verificação da derivada, a função exponencial de base $e$ e a função logaritmica de base $e$. Essa escolha foi fundamentada na perspectiva de que são funções de grande aplicabilidade nos diversos ramos da Engenharia. 
Vtilização de um software para a verificação da derivada de algumas funções de relativa complexidade de demonstração

Com a utilização do software Graphmatica, foram traçados os gráficos dessas funções e feitas as escolhas de pontos nos quais se traçaram as retas tangentes e, posteriormente, fizeram-se as determinações dos coeficientes angulares correspondentes.

A seguir, é apresentada a definição de derivada de uma função e aplicação dessa definição, utilizando-se o software Graphmatica.

\section{Definição de derivada}

A reta tangente à curva $y=f(x)$ em um ponto $P(a, f(a))$ é a reta que passa por $P$ com coeficiente angular $m$, tal que

$$
m=\lim _{x \rightarrow a} \frac{f(x)-f(a)}{x-a}
$$

desde que esse limite exista (STEWART, 2014, p. 131).

A derivada de uma função $f$ em $a$, denotada por $f^{\prime}(a)$, é

$$
f^{\prime}(a)=\lim _{h \rightarrow 0} \frac{f(a+h)-f(a)}{h}
$$

se o limite existir (STEWART, 2014, p. 133).

Se escrevermos $x=a+h$, então, $h=x-a$ e $h$ tendem a zero se, e somente se, $x$ tende a $a$. Consequentemente, uma maneira equivalente de enunciar a definição de derivada é: 
Utilização de um software para a verificação da derivada de algumas funções de relativa complexidade de demonstração

$$
f^{\prime}(a)=\lim _{x \rightarrow a} \frac{f(x)-f(a)}{x-a}
$$

Assim sendo, a reta tangente a $y=f(x)$ em $(a, f(a))$, é a reta que passa por $(a, f(a))$, cujo coeficiente angular é igual $f^{\prime}(a)$, ou seja, a derivada de $f$ em a (STEWART, 2014, p. 134).

\section{O Graphmatica}

O software Graphmatica é um gerador de gráficos de funções de uma variável nas suas várias formas: cartesiana, polar, paramétrica, logarítmica, trigonométrica, inequações e implícita. Com ele, ainda é possível gerar campos de vetores no plano e fornecer a solução das correspondentes equações diferenciais e, além disso, permite calcular: derivadas, integrais, máximos, mínimos e zeros de funções. Com o software Graphmatica podem-se construir vários gráficos em uma só tela, salvar informações e equações, bem como redimensionar as escalas em cada eixo. Como ferramenta adicional de Cálculo, o programa pode incluir símbolos de diferenciação, traçar retas tangentes a uma curva e calcular uma integral definida. 4

\section{Utilização do software Graphmatica}

Evidentemente, não se pode deixar de destacar a importância das deduções, utilizadas para se determinar a derivada de uma função. Po-

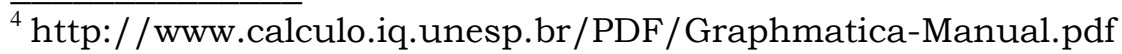


Vtilização de um software para a verificação da derivada de algumas funções de relativa complexidade de demonstração

rém, a determinação gráfica proposta nesse trabalho reforça os conceitos e definições apresentadas e pode fornecer ao aluno uma percepção de que os princípios de Cálculo e Geometria Analítica estão muito bem relacionados. Pelos conceitos apresentados, para se determinar a derivada de uma função $y=f(x)$ em um ponto de abscissa $x$, traça-se a reta tangente ao gráfico de $f(x)$ neste ponto e, a seguir, determina-se o coeficiente angular da reta tangente. O software Graphmatica permite essa determinação. Como exemplo, na figura 1 , mostram-se a reta tangente e o coeficiente angular da mesma, no ponto de abscissa 2 , para a função $\mathrm{y}=\mathrm{x}^{2}$.

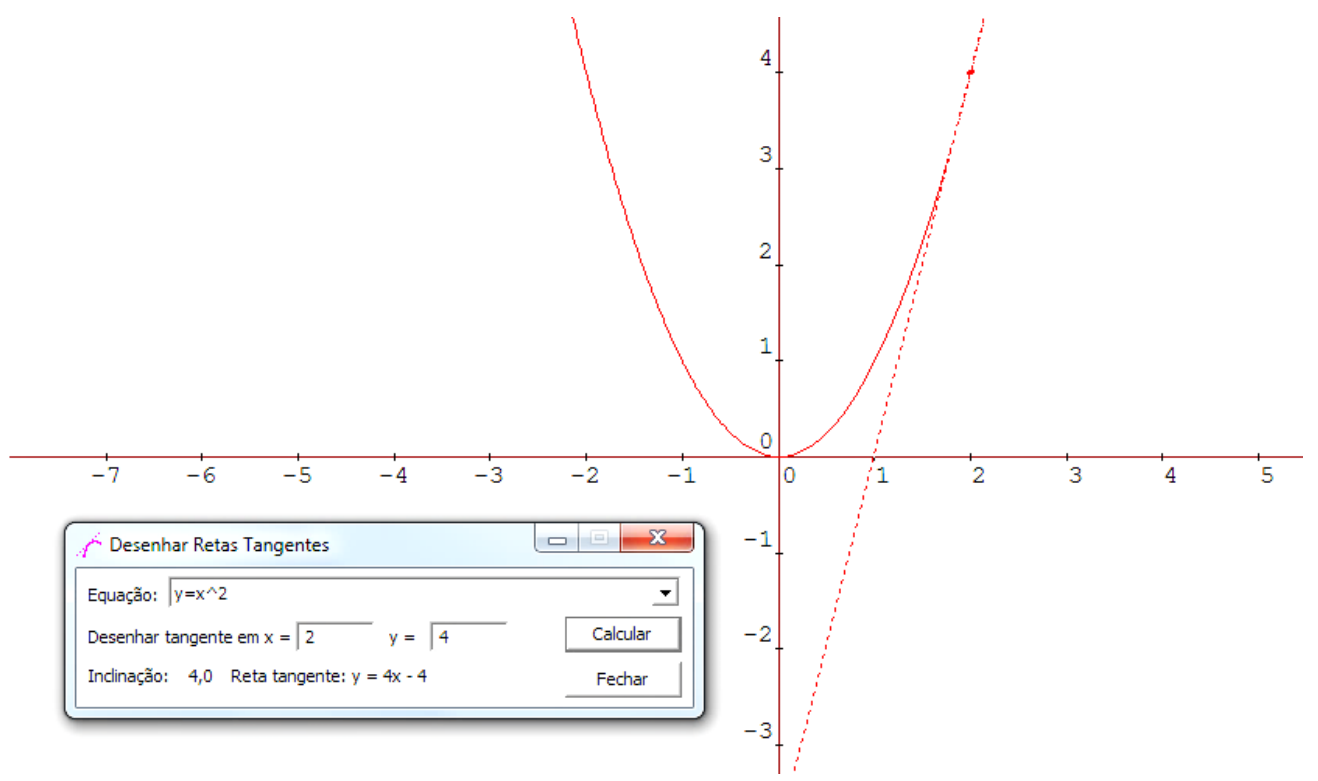

Figura 1- Gráfico da função $y=x^{2}$, a reta tangente no ponto de abscissa $2 \mathrm{e}$ o coeficiente angular da reta tangente $(m=4)$

Fonte: Elaborada pelos autores

Para a utilização do software Graphmatica, na verificação da derivada de algumas funções, serão adotados os seguintes procedimentos: 
Vtilização de um software para a verificação da derivada de algumas funções de relativa complexidade de demonstração

- Representa-se graficamente a função proposta.

- Escolhem-se alguns pontos do gráfico.

- Traça-se a reta tangente em cada um desses pontos.

- Anota-se o coeficiente angular de cada uma das retas tangentes.

- Traça-se o gráfico coeficiente angular da reta tangente versus abscissa $x$.

O gráfico obtido será aquele correspondente ao da função derivada da função dada inicialmente.

Utilizando-se os procedimentos apresentados, é mostrada, a seguir, a verificação das derivadas das funções citadas anteriormente.

\section{Derivada da função exponencial}

Seja a função $y=e^{2 x}$. Essa função, representada graficamente, figura 2, é:

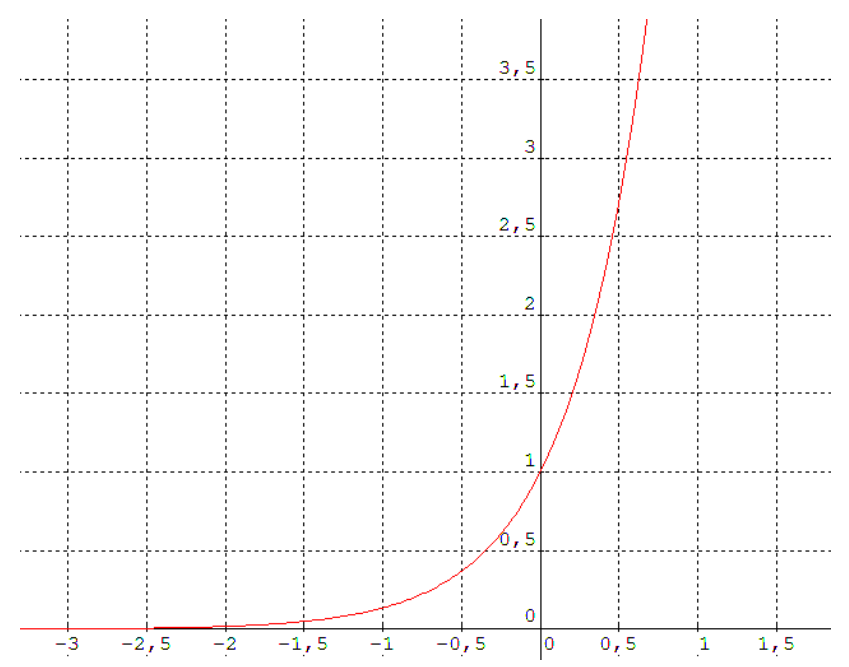

Figura 2 - Gráfico da função $y=e^{2 x}$

Fonte: Elaborada pelos autores 
Utilização de um software para a verificação da derivada de algumas funções de relativa complexidade de demonstração

Sejam considerados os pontos de abscissas $-1,5 ;-1,0 ;-0,5 ; 0,0 ; 0,5$; 1,$0 ; 1,5$. Os coeficientes angulares das retas tangentes em cada um desses pontos se encontram na tabela 1 .

Tabela 1- Coeficientes angulares (m) das retas tangentes aos pontos considerados

\begin{tabular}{|c|c|c|c|c|c|c|c|}
\hline Abscissa & $-1,5$ & $-1,0$ & $-0,5$ & 0,0 & 0,5 & 1,0 & 1,5 \\
\hline $\mathrm{m}$ & 0,0996 & 0,2707 & 0,7358 & 2,0000 & 5,4366 & 14,7781 & 40,1711 \\
\hline
\end{tabular}

Plotando-se os pontos da tabela 1 (figura 3), encontra-se:

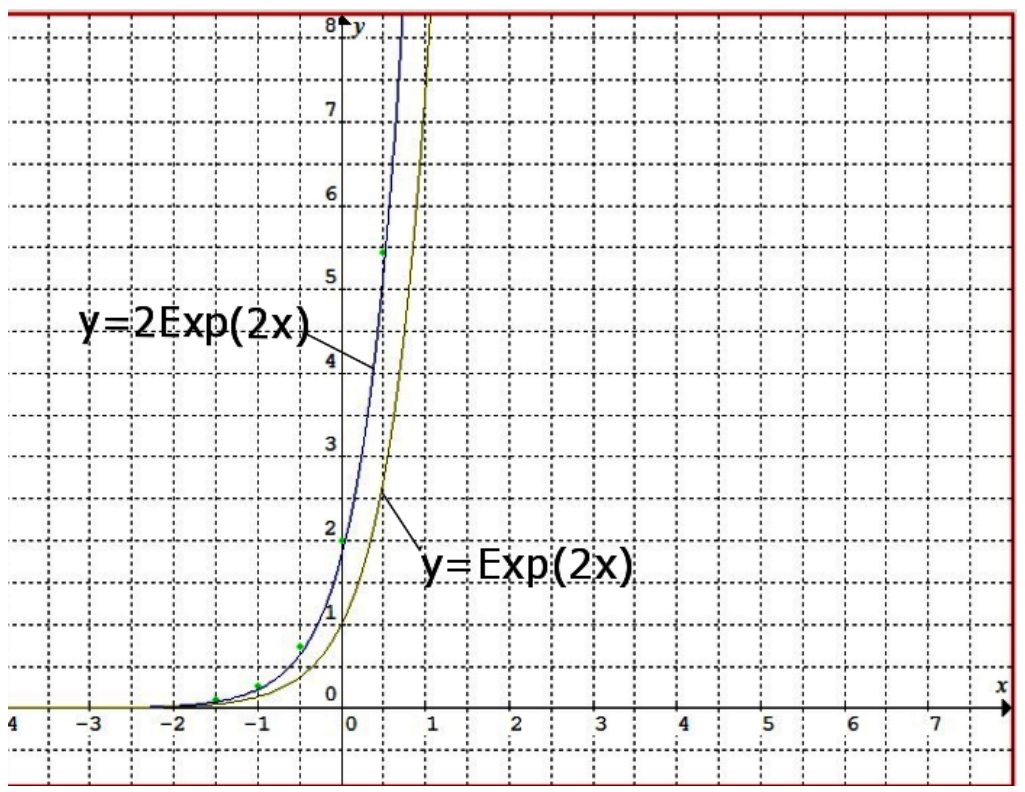

Figura 3 - Gráficos da função $y=e^{2 x}$ e da função derivada Fonte: Elaborada pelos autores 
Vtilização de um software para a verificação da derivada de algumas funçôes de relativa complexidade de demonstração

\section{Derivada da função logaritmica}

Considerando a função $y=\ln x$, pode-se também verificar graficamente, pelo processo já citado, a derivada dessa função que é $1 / x$. A demonstração dessa expressão derivada envolve aplicação das propriedades dos logaritmos e a utilização de um limite fundamental.

A figura 4 mostra, graficamente, a função proposta.

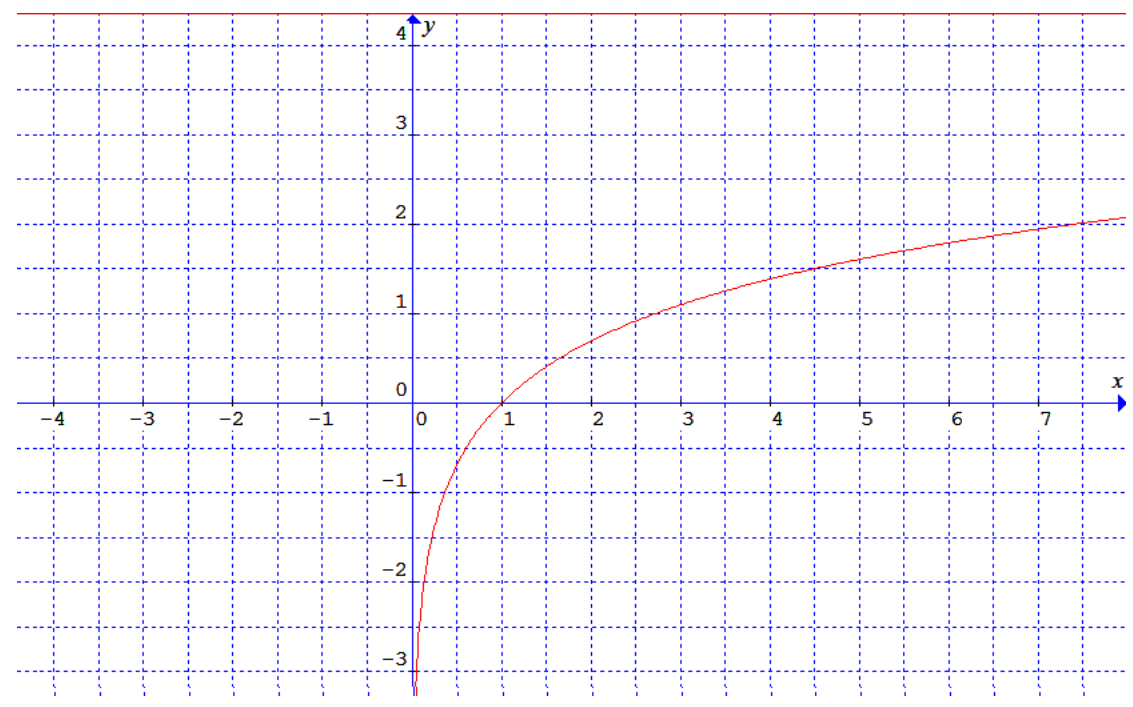

Figura 4 - Gráficos da função $y=\ln x$ Fonte: Elaborada pelos autores

Sejam considerados os pontos de abscissas 0,$15 ; 0,32 ; 0,50 ; 1,00$; 1,$50 ; 2,00 ; 2,50 ; 3,00 ; 3,50 ; 4,00 ; 4,50 ; 5,00$. Os coeficientes angulares das retas tangentes em cada um desses pontos se encontram na tabela 2 . 
Utilização de um software para a verificação da derivada de algumas funções de relativa complexidade de demonstração

Tabela 2- Coeficientes angulares das retas tangentes aos pontos considerados.

\begin{tabular}{|c|c|c|c|c|c|c|c|c|c|c|c|c|}
\hline Abscissa & 0,15 & 0,32 & 0,50 & 1,00 & 1,50 & 2,00 & 2,50 & 3,00 & 3,50 & 4,00 & 4,50 & 5,00 \\
\hline $\mathbf{m}$ & 6,67 & 3,13 & 2,00 & 1,00 & 0,67 & 0,50 & 0,40 & 0,33 & 0,29 & 0,25 & 0,22 & 0,20 \\
\hline
\end{tabular}

A figura 5 mostra os pontos plotados e a expressão matemática correspondente.

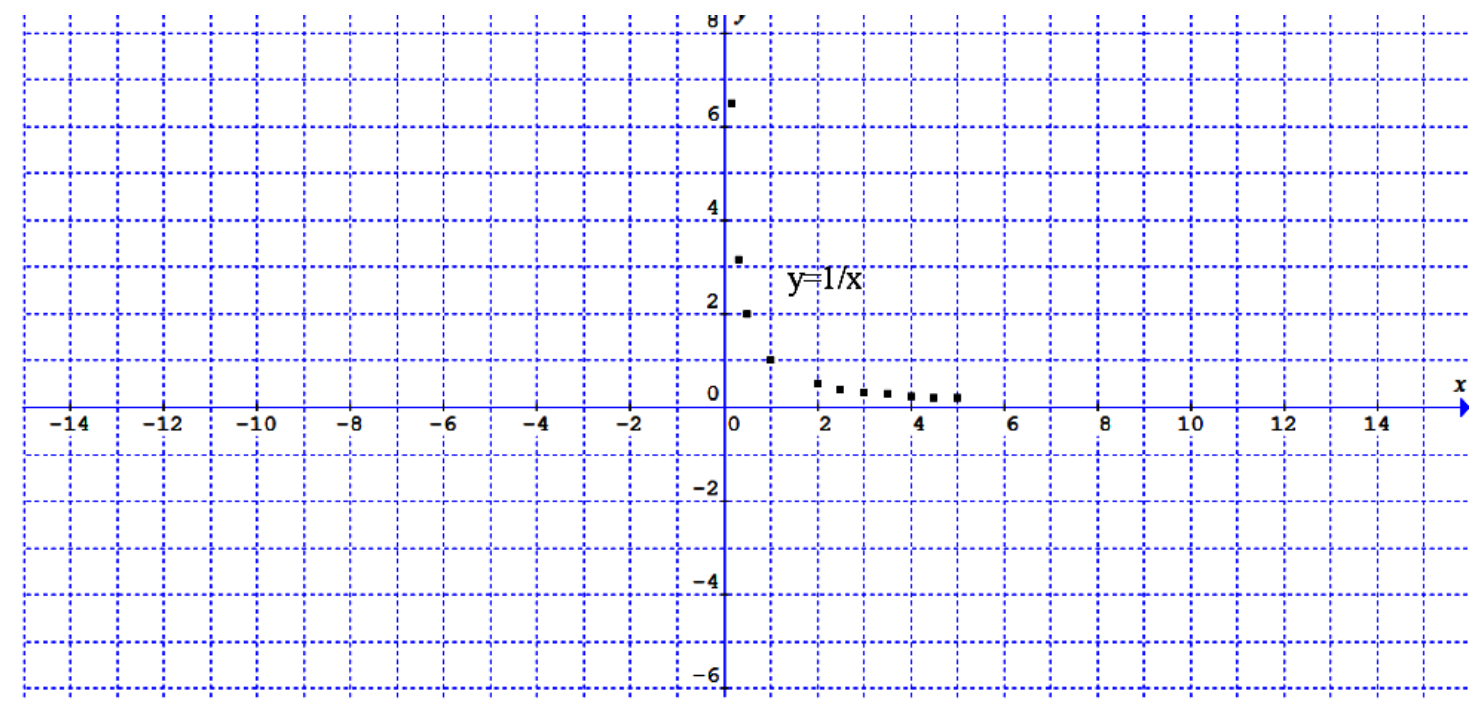

Figura 5 - Gráficos da derivada da função $y=\ln x$

Fonte: Elaborada pelos autores

Pode-se observar, nesses dois exemplos, que é bem simples a determinação da derivada de uma função, considerando-se vários pontos do gráfico dessa função. 
Vtilização de um software para a verificação da derivada de algumas funções de relativa complexidade de demonstração

\section{Análise dos resultados}

Ao ser determinado o coeficiente angular das retas tangentes aos pontos considerados nos gráficos, possibilitando a construção da tabela 1 para a função exponencial e a tabela 2 para a função logarítmica de base $e$, sendo conhecida a expressão da derivada, é possivel, a partir da definição, verificar a veracidade dessa expressão.

Para o caso da função exponencial $y=e^{2 x}$ cuja derivada é $y=2 e^{2 x}$, tem-se, por exemplo, para

$$
\begin{gathered}
x=1,5 \\
y^{\prime}(1.5)=2 \cdot e^{2 \cdot 1,5=40,1711}
\end{gathered}
$$

este é o valor do coeficiente angular da reta tangente ao ponto de abscissa 1,5 , verificado na tabela 1 . Dessa forma, os valores das derivadas nos diversos pontos da curva da função podem ser determinados diretamente a partir da aplicação da definição, utilizando-se os recursos do software. Ressalta-se o fato de que, a cada ponto escolhido, a definição de derivada é aplicada e verificada.

\section{Considerações finais}

A utilização de softwares educativos permite que os alunos tenham uma maior interação com os conceitos de Cálculo que são apresentados em sala de aula e que, na maioria das vezes, são abstratos e inibem, no caso de alguns, uma melhor compreensão do conteúdo. O aluno deve estar consciente de que o Cálculo é ferramenta importante e essa relevância se manifesta de modo especial para aqueles que pretendem se graduar em engenharia, uma vez que a vida profissional também exigirá vários 
Vtilização de um software para a verificação da derivada de algumas funções de relativa complexidade de demonstração

conceitos que serão aplicados nas várias situações do cotidiano profissional. Por outro lado, sempre é bom estar atento ao fato de que as explicações do professor em sala de aula são imprescindíveis. Isso significa que, no caso da proposta ora apresentada, o software Graphmatica é uma ferramenta adicional para complementar o trabalho do professor.

\section{Referências}

BARROS, Rodolfo Miranda de; MELONI, Luiz Geraldo Pedroso. O Processo de ensino e aprendizagem de Cálculo Diferencial e Integral por meio de metáforas e recursos multimídia. In: CONGRESSO BRASILEIRO DE ENSINO DE ENGENHARIA, 34., 2006, Passo Fundo. Anais... Passo Fundo: Ed. Universidade de Passo Fundo, Setembro de 2006.

BRONSON, Richard; COSTA, Gabriel. Equações Diferenciais. Coleção Schaum. 3. ed. Porto Alegre: Bookman, 2006.

CONCEIÇÃO, Katiani; GONÇALVES, Mírian B.. A resolução de problemas no processo ensino-aprendizagem de matemática nos cursos de engenharia. In: CONGRESSO BRASILEIRO DE ENSINO DE ENGENHARIA, 31., 2003, Rio de Janeiro. Anais... Rio de Janeiro: Associação Brasileira de Ensino de Engenharia, 2003.

EVES, Howard. Introdução à História da Matemática. Campinas: Editora Unicamp, 2007.

GONÇALVES, Mírian B.; ZUCHI, Ivanete. Investigação sobre os obstáculos de aprendizagem do conceito de limite. In: CONGRESSO BRASILEIRO DE ENSINO DE ENGENHARIA, 31., 2003, Rio de Janeiro. Anais... Rio de Janeiro: Associação Brasileira de Ensino de Engenharia, 2003. 
Utilização de um software para a verificação da derivada de algumas funções de relativa complexidade de demonstração

MIRANDA, Gustavo Alexandre de. Silvanus Phillips Thompson e a desmistificação do Cálculo: resgatando uma história esquecida. 2004. Dissertação (Mestrado em Educação Matemática) - Pontificia Universidade Católica, São Paulo. 2004.

REZENDE, Wanderley Moura. O Ensino de Cálculo: dificuldades de natureza epistemológica. 2003. Tese (Doutorado em Educação) - Faculdade de Educação, Universidade de São Paulo, 2003.

STEWART, James. Cálculo. São Paulo: CENGAGE Learning, 2014. v.1. 